\title{
Extended-Field Radiation Therapy
}

National Cancer Institute

\section{Source}

National Cancer Institute. Extended-Field Radiation Therapy. NCI Thesaurus. Code

C113811.

Radiation treatment delivered to large areas of the body, used primarily in patients with Hodgkin lymphoma. It involves the treatment of the lymph nodes that are affected by the disease, as well as the surrounding lymph node areas that the disease may have affected but is not clinically detected. 